\title{
A commentary on "Do patients with central sensitivity syndromes have poor subjective outcomes despite anatomical cure from pelvic organ prolapse surgery?"
}

\author{
Ghazaleh Rostaminia ${ }^{1}$ (D) \\ Received: 4 January 2021 / Accepted: 9 February 2021 / Published online: 23 February 2021 \\ (C) The International Urogynecological Association 2021
}

This prospective cohort study aimed to compare the outcomes of vaginal POP surgery between women with and without evidence of central sensitivity syndromes (CSS). Women who were scheduled for primary POP surgery during 2014 2017 were approached to participate in the study. Patients were categorized into those with or without CSS based on their score on the central sensitization inventory (CSI) questionnaire. All women had vaginal POP surgery without mesh including anterior repair, posterior repair and vaginal hysterectomy. Patients with concomitant urinary and/or fecal incontinence surgery or history of severe vaginal pain were excluded. The primary outcome was to compare the prolapse symptomology using the validated Pelvic Organ Prolapse Symptom Scale (POP-SS) scores, and the secondary outcomes were to compare the Patient Global Impression of Improvement (PGI-I) result, prolapse stage at the original site by the POPQ, subjective pain using McGill's Pain Questionnaire short form scores for somatic pain and satisfaction with surgery by using "EGGS" between the two groups 3-6 months after surgery.

Complete data were available for 62 patients, 23 patients had evidence of CSS, and 39 patients had no evidence of CSS. Women with evidence of CSS had statistically significantly higher pre- and postoperative POP-SS scores compared with women without CSS. Women with evidence of CSS had significantly more bothersome symptoms with less objective prolapse compared with women with no evidence of CSS. McGill's pain scores were higher in women with CSS both pre- and post-surgery.

Ninety-five percent of women without CSS achieved their goals and were satisfied with the surgery compared to $69.5 \%$ of women with CSS $(p<005)$. In terms of persistence of objective prolapse on POPQ (i.e., if the prolapse was at or beyond the hymen during maximum Valsalva maneuver), 2/13 (15\%) women in the CSS group had objective persistence of prolapse compared with $2 / 25(8 \%)$ in the group without CSS $(p<0.0005)$.

In summary, this study showed that women with evidence of CSS had less successful outcomes after POP surgery in terms of patient satisfaction, goal achievement and persistence of discomfort. These findings also demonstrated that, for patients with CSS, the subjective outcome is less favorable than in patients without CSS. While this study was limited by the small subject numbers and relatively short follow-up period, it brings attention to the value of complete history taking and physical examination during the initial evaluation. Identifying issues such as disturbed or unrefreshing sleep, symptoms of irritable bowel syndrome, difficulty with memory and concentration, and presence of widespread pain or myofascial trigger points during vaginal examination are strong markers of central sensitization syndrome, which might be a reason for persistent symptoms despite anatomical correction. Given the complexity of CSS and related symptoms, a biopsychosocial treatment approach has been accepted and should be offered for managing symptoms prior to scheduling any procedures.

Publisher's note Springer Nature remains neutral with regard to jurisdictional claims in published maps and institutional affiliations.
Ghazaleh Rostaminia

ghazalerostaminia@yahoo.com

1 NorthShore University HealthSystem, Skokie, IL, USA 